\title{
Contribution à l'amélioration de la qualité de prise en charge du paludisme au Centre de Santé Communautaire de Sanankoroba dans le district sanitaire de Kalabancoro, Mali
}

\section{Contribution to improving the quality of malaria care at the Sanankoroba Community Health Center in the health district of Kalabancoro, Mali}

\author{
Toungara $\mathrm{K}^{1}$, Coulibaly $\mathrm{MB}^{2}$, Koné $\mathrm{AK}^{3}$, Dolo $\mathrm{A}^{3}$, Minta $\mathrm{DK}^{3,4}$
}

1. Centre de santé communautaire de Sanancoroba (Bamako-Mali)

2. Centre de santé communautaire et universitaire de Konobougou (SégouMali)

3. Département d'Epidémiologie des Affections Parasitaires /Malaria Research and Training Center/Faculté de Médecine et d'Odontostomatologie/ Université des Sciences, des Techniques et des Technologies de Bamako, Mali

4. Service de maladie infectieuse au CHU du Point G (Bamako-Mali) *Auteur correspondant : Dr Mamadou Bayo COULIBALY, spécialiste en médecine de famille/médecine communautaire, Directeur technique du CSCom U de Konobougou, Chargé d'encadrement clinique D.E.S médecine de famille/médecine communautaire (Mali). bayo_coul@yahoo.fr ; Cel : 0022376067882

\section{Résumé}

Introduction : Le paludisme constitue un véritable problème de santé publique dans le monde et majeur dans les pays subsahariens. Sa prise en charge selon les recommandations de l'OMS n'est pas correctement effectuée partout. Le but de notre travail était de contribuer à l'amélioration de la qualité de la prise en charge du paludisme dans le centre de santé communautaire de Sanankoroba. Matériel et méthodes : II s'agissait d'une étude transversale à passage répété de recherche action de Susman. Elle s'est déroulée en quatre mois allant du 1ier juillet au 31 octobre 2019 dans le centre de santé de Sanankoroba. Ont été inclus dans cette étude les cas présumés ou confirmés du paludisme par les prestataires de santé du CSCom de Sanankoroba. Résultats : Après la mise en place du plan d'action entre l'évaluation initiale et finale, nous avons constaté une évolution de la concordance entre signes et classification du paludisme de $51 \%$ à 93,3\% (Chi2 = 40,848; $p<10^{-3}$ ). Le suivi et l'évolution appliqués par les prestataires étaient passés de $0 \%$ à $82,7 \%$ (Chi2 $=105,7 ; p=0,765$ ) au cours de l'évaluation finale. Les conseils sur les mesures de prévention de $0 \%$ à $81.3 \%$ après le plan d'action. Conclusion : Les insuffisances constatées au cours de cette étude ont été corrigées grâce à des planifications faites et leur mise en œuvre.

Mots clés : paludisme, centre de santé communautaire, Sanankoroba, Mali

\footnotetext{
Abstract

Introduction: Malaria is a real public health problem worldwide and a major one in sub-Saharan countries. Its management according to WHO recommendations is not correctly carried out everywhere. The purpose of our work was to contribute to improve the quality of malaria management in the Sanankoroba community health center. Methods: This was a repeat cross-sectional action research study by Susman. It took place in four months
}

from July 1 to October 31, 2019 in the Sanankoroba health center. Included patients in this study were suspected or confirmed cases of malaria by health providers from the CSCom de Sanankoroba. Results: After the implementation of the action plan between the initial and final evaluation, we noted an evolution in the agreement between signs and classification of malaria from $51 \%$ to 93.3\% (chi2 = 40,848; $p<10^{-3}$ ). The monitoring and evolution applied by providers had gone from $0 \%$ to $82.7 \%$ (chi2 $=105,7 ; p=0,765$ ) during the final evaluation. Advice on prevention measures gone from $0 \%$ to $81.3 \%$ after the action plan. Conclusion: The shortcomings noted during this study were corrected due to the planning made and its implementation.

Keywords: malaria, community health center, Sanankoroba, Mali

\section{INTRODUCTION}

Le paludisme constitue un véritable problème de santé publique dans le monde $(1,2)$ et majeur dans les pays subsahariens $(3,4)$. Le paludisme est une maladie infectieuse tropicale potentiellement mortelle causée par un parasite qui infecte les érythrocytes (5).

Pour les personnes touchées, la combinaison d'un diagnostic manqué ou retardé avec le taux de mortalité élevé d'infection à Plasmodium falciparum peut entraîner des conséquences graves (6). L'OMS estime que 3,3 milliards de personnes sont à risque d'infection par le paludisme dont 1,2 milliard sont à haut risque, principalement en Afrique (49\%). II a des conséquences économiques graves. En effet, il consomme 40\% des dépenses de santé publique en Afrique ; il entrave la scolarité des enfants et, ainsi, le développement social (7). Sur les 91 pays signalant des cas de paludisme indigènes, 15 pays supportaient $80 \%$ de la charge mondiale (8). En 2010, selon l'Organisation mondiale de la santé (OMS), 90\% des 216 millions de cas estimés et les 655 000 décès sont survenus en Afrique subsaharienne, principalement chez les enfants de moins de 5 ans $(1,9)$. Près de $66 \%$ de ces décès impliquaient des enfants de moins de cinq ans, une population particulièrement vulnérable à cette maladie (8).

Au Mali, le paludisme est un problème majeur de santé publique, car il représente 34 à $39 \%$ des motifs de consultations dans les services de santé (1)

Pour faire face à cette endémie avec ses partenaires, le Mali, à travers la politique nationale de lutte contre le paludisme, a entrepris la mise à échelle des mesures de lutte efficaces qui sont entre autres : la confirmation du diagnostic de paludisme et un traitement précoce et 
approprié des cas confirmés, l'utilisation de moustiquaire imprégnée d'insecticide à longue durée d'action (MILD), le traitement préventif intermittent (TPI) à la SulfadoxinePyriméthamine (SP) chez la femme enceinte, la Chimio Prévention du Paludisme Saisonnier de l'enfant (CPSe) avec la Sulfadoxine-Pyriméthamine (SP) et l'Amodiaquine $(A Q)$, et la pulvérisation intra domiciliaire (PID) (10) .

Lors des supervisions, les insuffisances ont été identifiées au cours du deuxième trimestre 2018 dans la confirmation du diagnostic de paludisme et un traitement précoce et approprié des cas confirmés. Ces insuffisances peuvent entraver la qualité des soins offerts dans le cadre de la prise en charge du paludisme. II s'agit de problème d'ordre technique et de ressources. Au regard des faiblesses, et soucieux des conséquences qu'elles peuvent engendrer, nous avons mené cette étude qui avait pour but de contribuer à l'amélioration de la qualité de la prise en charge du paludisme dans le centre de santé communautaire de Sanankoroba.

\section{MATERIEL ET METHODES :}

II s'agissait d'une étude transversale à deux passages, de recherche action de Susman (11). L'étude s'est déroulée pendant une période de quatre mois allant du 1ier juillet au 31 octobre 2019 dans le CSCom de Sanankoroba dans le district sanitaire de Kalabancoro/Région de Koulikoro.

Ont été inclus dans l'étude, les cas présumés ou confirmés du paludisme par les prestataires de santé du CSCom de Sanankoroba. N'ont pas été inclus dans cette étude, les autres pathologies prises en charge par le personnel du CSCom.

Lors de la première et de la seconde évaluation de l'étude, nous avons obtenu soixante-quinze cas ou suspects de paludisme simple comme taille de l'échantillon. Le questionnaire élaboré nous a servi de support pour la collecte des informations dans les registres de consultations et de laboratoire. Nous avons également fait l'observation directe du personnel à travers une grille. Les données ont été collectées avec le questionnaire, tout en procédant à l'exploitation des enregistrements de tous les cas suspects du paludisme, suivi des questions d'éclaircissement. Les données ont été saisies et analysées sur Epi Info version 7. Le test de Chi2 a été utilisé pour comparer des proportions.

Dans un premier temps, nous avons fait une évaluation initiale afin de connaitre les points forts, les insuffisances et les goulots d'étranglements. Dans un deuxième temps, nous avons mis en œuvre le plan d'action que nous avons élaboré avec le personnel et les membres de l'Association de Santé Communautaire (ASACO) après une formation et sensibilisation des différents intervenants. Enfin, une évaluation finale a été réalisée pour voir l'effet du plan d'action.

\section{RESULTATS}

Locaux et personnel

Notre étude transversale à passage répété de recherche action s'est déroulée au CSCom de Sanankoroba du mois d'août à octobre 2019 sur l'amélioration de la qualité de prise en charge du paludisme.

Au cours de cette étude, nous avons constaté l'existence des salles de consultations appropriées au niveau du dispensaire, de la maternité et un laboratoire pour les analyses.

Les consultations étaient assurées tous les jours par douze agents dont huit qualifiés soit $66,67 \%$ et quatre non qualifiés (tableau I). Ces agents ont été formés sur la prise en charge du paludisme.

Disponibilité des médicaments, intrants et consommables (figure 1)

Durant la période d'étude, nous n'avons pas connu de rupture de médicaments contre le paludisme, des consommables et des intrants qui sont le TDR et ceux de la goutte épaisse.

\section{Utilisation du protocole}

Les protocoles de traitement sur les différentes molécules étaient affichés et la disponibilité du module sur les normes et protocoles du Mali révisé 2016 de prise en charge du paludisme dans les structures sanitaires. A l'évaluation primaire, l'utilisation du protocole était à $51 \%$ tandis qu'elle était à $93 \%$ à l'évaluation finale de l'enquête (tableau II).

La qualité du diagnostic clinique du paludisme:

Nous avons constaté au cours des deux évaluations que l'accueil était bien jusqu'à 98,7\%.

A l'enquête primaire, la recherche des signes de complication ne se faisait pas tandis qu'elle était à $77,3 \%$ à l'enquête finale, Chi2 = 94,565; $p<10^{-3}$.

L'effectivité de l'examen physique était de $69,3 \%$ à l'évaluation initiale et $97,3 \%$ pendant l'évaluation finale, Chi2 = 21,168; $p<10^{-3}$.

Au début, la concordance entre signes et classification du paludisme était à $51 \%$ et après la mise en place du plan d'action 93,3\% ont été retrouvés, Chi2 = 40,848; $p<10^{-3}$.

\section{Qualités du diagnostic biologique}

Au début de notre étude, les prestataires ne portaient pas le gant lors des prélèvements. Après la mise en œuvre du plan action, le port de gant par les prestataires est revenu à $73.3 \%$, Chi2 $=68,842 ; p<10^{-3}$.

Seulement $29,33 \%$ des prestataires nettoyaient le point d'injection du début contre $85,3 \%$ à la fin de l'évaluation, Chi2 $=35,032 ; p<10^{-3}$.

L'utilisation correcte de TDR était plus $80 \%$ durant les deux évaluations respectivement $84,6 \%$ et $86,5 \%$, Chi2 = 5,$635 ; p=0,018$.

Les prestataires respectaient à $67,5 \%$ le temps de lecture à l'évaluation finale contre $0 \%$ au début de l'évaluation, Chi2 $=35,755 ; p<10^{-3}$.

La qualité traitement du paludisme :

Nous n'avons pas noté de différence majeure au cours des deux évaluations dans la concordance entre classification et traitement lors de la prise en charge du paludisme qui sont $89,3 \%$ et $92 \%$, Chi2 $=0,089 ; p=0,765$ (tableau III). 
Le suivi et l'évolution appliqués par les prestataires ont passé de $0 \%$ de l'évaluation initiale à $82,7 \%$ au cours de l'évaluation finale, Chi2 = 105,7; $p=0,765$ (tableau IV). Les conseils sur les mesures de prévention n'étaient pas du tout faits avant soit $0 \%$ contre $81,3 \%$ après l'intervention.

\section{DISCUSSION}

Locaux et personnel : Au cours de cette étude, nous avons constaté l'existence des salles de consultations appropriées au niveau du dispensaire, de la maternité et un laboratoire pour les analyses.

Les consultations étaient assurées tous les jours par douze agents dont les deux tiers étaient qualifiés, tous formés sur la prise en charge du paludisme (tableau I).

Disponibilité des médicaments, intrants et consommables

Durant la période d'étude nous n'avons pas connu de rupture de médicaments, de consommables et d'intrants. Tous les approvisionnements ont été faits au district sanitaire de Kalabancoro sauf les intrants du laboratoire (figure 1).

\section{Utilisation du protocole}

Les protocoles de traitement sur les différentes molécules étaient affichés et la disponibilité du module sur les normes et protocoles révisé 2016 de prise en charge du paludisme dans les structures sanitaires. Nous disions que cela facilitait la prise en charge et le respect des normes. Selon SOW MS et al., la disponibilité et l'utilisation du protocole national de prise en charge du paludisme simple restent faibles ; la formation continue du personnel semble nécessaire (12).

L'utilisation du protocole a significativement évoluée entre l'évaluation primaire et finale. L'utilisation du protocole pourrait permettre de réduire les erreurs et d'avoir le résultat escompté. Plus de la moitié $(60,2 \%)$ des prescriptions étaient conformes au protocole, mais aucun prestataire ne s'en servait parce qu'inaccessible et seulement $72 \%$ savaient son existence dans l'étude de SOW MS et al. (12).

La qualité du diagnostic clinique du paludisme :

Nous avons constaté au cours des deux évaluations que la quasi totalité des accueils était bien. Notre résultat était semblable à celui de Dakoua $\mathrm{F}$ et al. qui avait eu $100 \%$ de satisfaction à l'accueil dans leur évaluation finale (13). Cela favorise la mise en place d'une confiance et donc une bonne collaboration qui facilitera l'adhésion au traitement et au suivi.

A l'enquête primaire, la recherche des signes de complication ne se faisait pas tandis qu'elle était à $77,3 \%$ à la finale. La recherche de signes de complications est capitale car pourrait dicter la conduite à tenir. Cependant, dans l'étude de Coulibaly $C$ et al., la recherche des signes mineurs et de gravité associés à la fièvre était systématique (1)

L'effectivité de l'examen physique a beaucoup évolué entre les deux évaluations du tiers environ à près de $100 \%$. La qualité de l'anamnèse et de l'examen physique commande les étapes suivantes de l'évaluation du patient et guide les choix dans la masse initialement déroutante des examens complémentaires ; l'anamnèse et l'examen physique complets constituent les fondements de l'évaluation clinique selon Bates B (14).

Au début, la concordance entre signes et classification du paludisme était à $51 \%$ et à $93,3 \%$ après la mise en place du plan d'action (tableau II, III). Nous disons que la classification des cas de paludisme est nécessaire car c'est en fonction de cela que le traitement est donné. Biga $\mathrm{N}$ disait dans ses travaux qu'en cas d'absence de concordance, et en cas de difficulté diagnostique, le recours à des explorations complémentaires plus sophistiquées prend toute sa valeur (15).

Au total nous avons noté une bonne évolution de la qualité du diagnostic clinique du paludisme de la phase initiale à la phase finale (tableau IV).

Pour assurer cette qualité, il fallait l'effectivité correcte des actes qui sont : l'accueil, la recherche des signes de complication, l'examen physique, la concordance entre signes et classification et classification et traitement.

\section{Qualités du diagnostic biologique}

Au début de notre étude les prestataires ne portaient pas de gants lors des prélèvements. Après la mise en œuvre du plan le port de gants est revenu à $73,3 \%$.

L'utilisation correcte de TDR était de plus $50 \%$ durant les deux évaluations. Pour Faye SL, les TDR ont une utilité épidémiologique reconnue ; en revanche, leur intégration positive requiert un changement de comportements que les soignants et les bénéficiaires ne sont pas toujours prêts à assumer (16). En révanche, Yaméogo TM et al. avaient utilisé surtout la goutte épaisse à $55,1 \%$ (17). Pour la majorité des laboratoires d'analyses médicales impliqués dans le diagnostic du paludisme d'importation, les TDR associés au frottis mince sont une solution suffisamment sensible et robuste (18). Pour autant, un résultat négatif ne doit jamais faire écarter définitivement un accès palustre évolutif ; aussi, en cas de doute ou d'absence de diagnostic, il ne faut pas hésiter à refaire un prélèvement 12 à 24 heures plus tard (18).

Les deux tiers des prestataires respectaient le temps de lecture du TDR à l'évaluation finale contre aucun au début de l'évaluation. Le respect du temps de lecture pourrait réduire les cas de faux négatif ou positif.

L'accomplissement de la qualité du diagnostic biologique nécessite la vérification de la date de péremption, le port de gant, nettoyage du point d'injection, l'utilisation correcte de TDR et le respect du temps de lecture.

La qualité traitement du paludisme :

Nous n'avons pas noté de différence majeure au cours des deux évaluations dans la concordance entre classification et traitement lors de la prise en charge du paludisme. Sur les 165 cas présumés, le traitement était approprié dans $88,5 \%$ des cas pour Yaméogo TM et al (17), similaire à notre résultat. Notre résultat était supérieur à celui d'Elmansouf $L$ et al dont seulement $32 \%$ des prises en charge étaient conformes aux recommandations de 1999 ; pour eux l'application 
optimale des recommandations aurait permis un gain de 117 jours d'hospitalisation, soit $54000 €$ (19). Au terme de l'analyse, $63,3 \%$ des prescriptions étaient conformes au protocole (20) qui était aussi inférieur au nôtre. Le suivi et l'évolution appliqués par les prestataires a largement évolué. A l'étude finale, notre résultat était un peu supérieur à celui de Coulibaly $C$ et al. chez qui, la majorité des patients reçus $(71,5 \%)$ avait bénéficié d'un rendezvous pour le suivi, ainsi que des questions de compréhension sur la poursuite du traitement à domicile (1). Bouchaud $O$ et al. avaient dit que le suivi est très important pour s'assurer, pendant la phase critique des premiers jours, de la bonne évolution immédiate de l'accès palustre et de la tolérance de l'antipaludique, et, à plus long terme, de l'absence de rechute (9).

Les conseils sur les mesures de prévention n'étaient pas du tout faits avant contre $81,3 \%$ après l'intervention (tableau V). Alors que pour Kouassi DP et al., en pratique, seulement $4,76 \%$ utilisait comme méthode de prévention du paludisme la moustiquaire imprégnée et 2,93\% pratiquaient l'hygiène environnementale (21). Les mesures préventives ne sont pas difficiles avec la disponibilité des moustiquaires. Quand ces mesures ne sont pas prodiguées, on peut se réinfecter très facilement. Pour l'acquisition de cette qualité, il fallait assurer la concordance entre classification et traitement, le suivi, l'évolution et les conseils sur les mesures de prévention lors de la prise en charge du paludisme.

Points forts

> La disponibilité de ressources humaines formées et leur implication ;

- La disponibilité des deux tests, des médicaments et des consommables :

$>$ L'implication de l'ASACO :

> L'atteinte des objectifs de plus de $50 \%$;

\section{Points à améliorer}

$>$ L'insuffisance dans le port de gants lors des prélèvements ;

$>$ L'insuffisance dans la recherche des signes de complication.

\section{CONCLUSION :}

Au Mali, le paludisme demeure un problème majeur de santé publique de par sa morbidité, sa mortalité et les répercussions socio-économiques qu'il occasionne. Pour faire face à cette endémie avec ses partenaires, le Mali à travers la politique nationale de lutte contre le paludisme a entrepris la mise à échelle des mesures de lutte efficaces dont l'application des dimensions de la prise en charge dans les structures sanitaires est essentielle.

Au cours de cette étude, nous avons constaté des insuffisances qui ont été analysées. C'est après que nous avons fait des planifications dans la résolution des problèmes constatés.

Après l'implication de tous les acteurs, nous avons pu faire face aux goulots. Des propositions de solutions ont été faites pour la pérennité et la consolidation des acquis. II s'agissait pour le Directeur technique du centre : de faire les supervisions régulièrement au CSCom ; et pour l'équipe technique du CSCom de : faire le port systématique de gants pour les prélèvements et faire la recherche des signes de complication lors de la prise en charge du paludisme.

\section{REFERENCES}

1. Coulibaly C, Fomba S, Sangho H, K A Sidibé, Touré K, K H Dembélé : Prise en charge des cas de paludisme chez les enfants de 0 à 5 ans et perception des mères dans un service de pédiatrie à Bamako. MALI MEDICAL. 2012 ; XXVII : 1-6.

2. Seck I, Fall IS, Faye A, Ba O, Tal-Dia A : Connaissances, attitudes et pratiques des femmes sur le paludisme, dans la zone rurale de Poponguine, Sénégal. Médecine Tropicale. 2008 ; 68 (6) : 629-633.

3. Salissou A, Halima BM, Abani M, Adehossi E, Daou M, Boureima S, Maimouna H, Ibrahim M.L : Efficacité et tolérance de l'association artemether lumefantrine dans le traitement du paludisme simple a Plasmodium falciparum AU NIGER. J. Rech. Sci. Univ. Lomé (Togo), 2012, Série D, 14(1) : 79-84.

4. Nagalo K, Dao F, Minodier P, Sawadogo O, Sanon H, Tall $\mathbf{F} \mathbf{H}$, Diarra $\mathbf{Y}$ : Le paludisme congénital maladie à Plasmodium falciparum : aspects épidémiologiques, cliniques, biologiques, thérapeutiques et pronostiques à Ouagadougou, Burkina Faso. Pan African Medical Journal $2014 ; 18: 47$, P 2-7.

5. Houzé S: [Malaria: immuno-permissive management in the prevention of transfusional malaria]. Transfus Clin Biol. 2019; 26(3) :192-194.

6. Zoller T, Naucke TJ, May J, Hoffmeister B, Flick H, Williams $\mathrm{CJ}$, et al. Malaria transmission in non-endemic areas: case report, review of the literature and implications for public health management. Malar J. 2009 ; 8: 21.

7. Faye A, Ndiaye P, Camara M D, Badiane O, Wone I, Diongue M, Seck I, Dia A T et Dia A L : Evaluation économique des tests de diagnostic rapide dans le traitement du paludisme. Santé Publique. 2010 ; 22 : 617 à 623 .

8. Pradines B, Robert MG: [Current situation of malaria in the world]. Rev Prat. $2019 ; 69(2): 146-149$.

9. Bouchaud O, Pull L, Siriez J Y : Le paludisme simple en 2012 : grands classiques et nouveautés. La Lettre de l'Infectiologue. 2012• Tome XXVII - n 6 ; 227-232.

10. Programme de lutte contre le paludisme : Plan Stratégique De Lutte Contre Le Paludisme 2013-2017. 2013. consulté le 05/09/2018.Available from http://mail.cnom.sante.gov.ml/docs/PStrag\%20201317PN LP.pdf

11. Gabriel G, Lessard-Hébert M : La recherche action : Ses fonctions, son fondement et son instrumentalisation. Presse de l'Université de Québec. 1987 ; 125p.

12. SOW M S, DIALLO M O S, BOUSHAB B $M$, SAVADOGO M, DIAWARA I $\mathrm{K}$ : Evaluation de l'utilisation du protocole national de prise en charge du 
paludisme simple des enfants à Matam, Guinée Conakry. PAN AFRICAN MEDICAL JOURNAL $2016 ; 23$.

13. Dakouo F,Fomba S, Coulibaly M. B : Analyse des indicateurs du processus de gestion d'un centre de premier niveau : Cas du CSCOM de Konobougou, Mali. Jaccr Africa. 2019 ; 3(3) ; 250-259.

14. Barbara Bates : Guide de l'examen clinique. Paris, 7ième édition française, 2014.

15. Biga $N$ : Examen clinique du pied et du cou de pied. Collecte des données et construction d'enchaînements étiopathogéniques. Revue de chirurgie orthopédique et traumatologique. 2009 ; 95 : 2009, 47-54.

16. Faye SL: Améliorer la prise en charge du paludisme par les tests de diagnostic rapide (TDR) : appropriation par les prestataires et bénéficiaires de soins au Sénégal. Bulletin de la Société de pathologie exotique. 2012 ; 105 : 237244.

17. Yaméogo TM, Kyelem CG, Ouédraogo SM, DIALLO 0 J, Moyenga L, Poda G E A et Guiguemdé T R : Diagnostic et prise en charge du paludisme grave chez l'adulte : observance des directives nationales au Burkina Faso. Bulletin de la Société de pathologie exotique. 2011 ; 104 : 284-287.

18. Berry A, Iriart X, Magnaval JF : Nouvelles méthodes de diagnostic du paludisme. Revue francophone des laboratoires. 2009 ; 2009 : Pages 65-70.

19. Elmansouf L, Dubos F, Dauriac A, Courouble C, Pruvost I, Dervaux B : Évaluation des pratiques dans la prise en charge du paludisme d'importation de l'enfant dans la région Nord-Pas-de-Calais. Médecine et maladies et maladies infectieuses. $2011 ; 41: 145-151$.

20. M Nzayirambah $\mathbf{0}$, Freund RJ, Millet $\mathbf{P}$, Lombrail $\mathbf{P}$, Malvy D, Potel G : Évaluation de l'application du protocole national de traitement du paludisme simple dans les formations sanitaires de la ville de Kigali, Rwanda Médecine et maladies infectieuses. 2008 ; 38 : 119-124.

21. Kouassi DP, Dagnan S : Connaissances, attitudes et pratiques des ménages en matière de prévention du paludisme : cas d'un quartier précaire d'Abidjan, côte d'ivoire. cah. Santé publique, $2012 ; 11: 66-72$.

\section{LISTE DES TABLEAUX ET FIGURES}

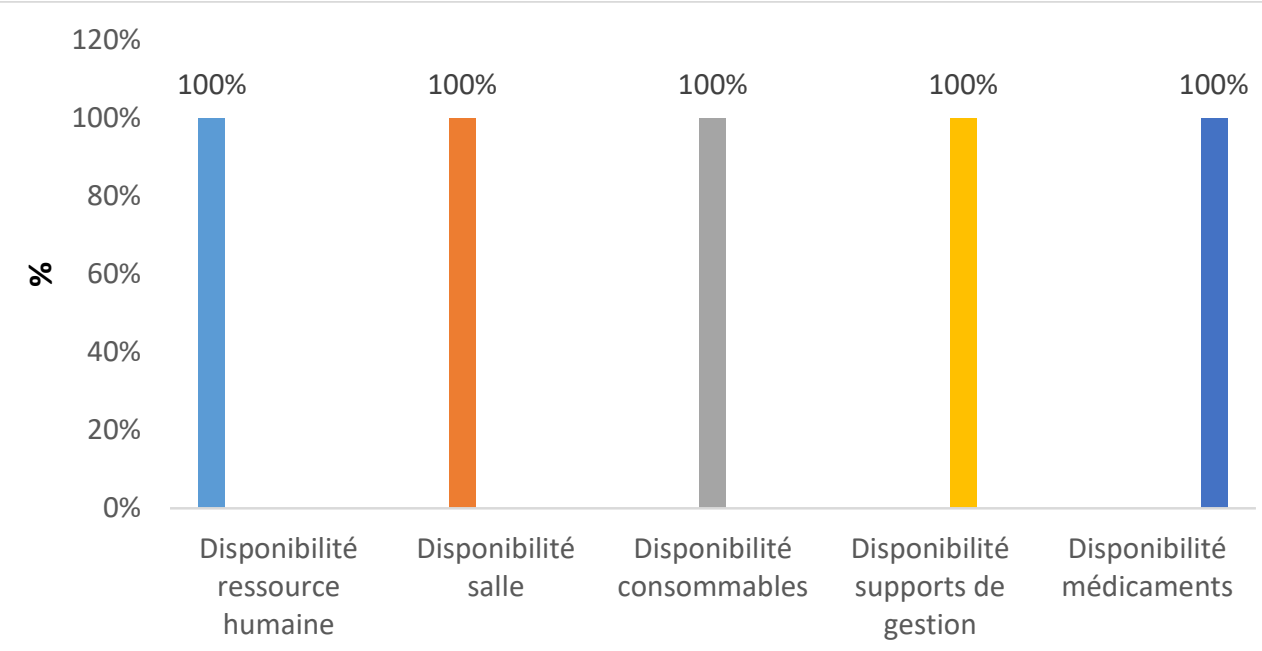

Disponibilité des éléments de prise en charge du paludisme

Figure 1 : La disponibilité des différents éléments rentrant dans la prise en charge du paludisme Tous les éléments étaient disponibles à $100 \%$.

Tableau I : Relatif au personnel

\begin{tabular}{|l|c|c|}
\hline Personnel qualifié & Effectif & $\%$ \\
\hline Oui & 8 & 68 \\
\hline Non & 4 & 32 \\
\hline Total & 12 & 100 \\
\hline
\end{tabular}

Dans $68 \%$ des cas, le personnel qualifié était disponible. 
Tableau II : Concordance entre signes et classification lors de la prise en charge du paludisme. On a constaté une augmentation de $42,3 \%$ après l'intervention.

\begin{tabular}{lcc|ccc}
\hline \multicolumn{2}{c}{ Avant } & & \multicolumn{2}{c}{ Après } \\
\hline & Effectif & $\%$ & & Effectif & $\%$ \\
Oui & 38 & 51,0 & Oui & 70 & 93,3 \\
Non & 37 & 48,0 & Non & 5 & 6,7 \\
TOTAL & 75 & 100,0 & TOTAL & 75 & 100,0 \\
\hline
\end{tabular}

Nous avons constaté une évolution de la concordance entre les signes et la prise en charge de $51 \%$ à $93,3 \%$.

Tableau III : Concordance entre classification et traitement lors de la prise en charge du paludisme

\begin{tabular}{lcc|ccc}
\hline \multicolumn{7}{c|}{ Avant } & & Après & & \\
\hline & Effectif & $\%$ & & Effectif & 9 \\
Oui & 67 & 89,3 & Oui & 69 & 92,0 \\
Non & 8 & 10,7 & Non & 7 & 8,0 \\
TOTAL & 75 & 100,0 & TOTAL & 75 & 100,0 \\
\hline
\end{tabular}

Seulement dans $10,7 \%$ des cas il n'y avait pas de concordance contre $8 \%$ après l'intervention.

Tableau IV : Relatif au suivi et à l'évolution lors de la prise en charge du paludisme

\begin{tabular}{lcc|ccc}
\hline & \multicolumn{2}{c|}{ Avant } & \multicolumn{3}{c}{ Après } \\
\hline & Effectif & $\%$ & & Effectif & $\%$ \\
Fait & 0 & 0,0 & Fait & 62 & 82,7 \\
Non fait & 75 & 100,0 & Non fait & 13 & 17,3 \\
TOTAL & 75 & 100,0 & TOTAL & 75 & 100,0 \\
\hline
\end{tabular}

On a observé une augmentation de $82,7 \%$ après le plan d'action.

Tableau V : Conseils sur les mesures prévention lors de la prise en charge du paludisme

\begin{tabular}{lcc|ccc}
\hline & Avant & & \multicolumn{3}{c}{ Après } \\
\hline & Effectif & $\%$ & & Effectif & $\%$ \\
Fait & 0 & 0,0 & Fait & 61 & 81,3 \\
Non fait & 75 & 100,0 & Non fait & 14 & 18,7 \\
TOTAL & 75 & 100,0 & TOTAL & 75 & 100,0 \\
\hline
\end{tabular}

Les conseils sur les mesures prévention n'étaient pas donnés du tout au début contre $81,3 \%$ à la fin à l'évaluation finale. 